\title{
CURVATURES AND SIMILARITY OF OPERATORS WITH HOLOMORPHIC EIGENVECTORS
}

\author{
MITSURU UCHIYAMA
}

\begin{abstract}
The curvature of the holomorphic vector bundle generated by eigenvectors of operators is estimated, and the necessary and sufficient conditions for contractions to be similar or quasi-similar with unilateral shifts are given.
\end{abstract}

\section{INTRODUCTION}

Let $H$ be a separable complex Hilbert space, $g_{r}(n, H)$ the set of all $n$ dimensional subspaces of $H$, and $\gamma$ a mapping from an open connected set $\Omega$ in the complex plane $\mathbf{C}$ to $g_{r}(n, H)$. Then $\gamma$ is called a holomorphic curve over $\Omega$, if for each $w_{0}$ in $\Omega$, there is a nbhd $\Delta$ of $w_{0}$ and vector valued holomorphic functions $\gamma_{i w}$ on $\Delta(i=1, \ldots, n)$ satisfying $\gamma_{w}=\bigvee\left\{\gamma_{i w}: i=\right.$ $1, \ldots, n\}$ for $w$ in $\Delta$. In this case, the Hermitian holomorphic vector bundle $\left(E_{\gamma}, \Omega, \pi\right)$ is defined as

$$
E_{\gamma}=\left\{(x, w) \in H \times \Omega: x \text { in } \gamma_{w}\right\}, \quad \pi(x, w)=w,
$$

and hence for this bundle, the canonical connection and curvature $\mathscr{K}_{\gamma}$ are well defined [19]. We call $\gamma_{1 w}, \ldots, \gamma_{n w}$ a frame for $E_{\gamma}$ on $\Delta$. The matrix form of $\mathscr{K}_{\gamma}(w)$ with respect to the above frame is

$$
-\frac{\partial}{\partial \bar{w}}\left(G \gamma^{-1} \frac{\partial G \gamma}{\partial w}\right)
$$

where $G_{\gamma}(w)$ is the Gram matrix whose $(i, j)$ component is $\left(\gamma_{j}(w), \gamma_{i}(w)\right)$ (cf. [4]).

In case of $n=1$, we have especially

$$
\mathscr{K}_{\gamma}(w)=-\frac{\partial^{2}}{\partial w \partial \bar{w}} \log \left\|\gamma_{1 w}\right\|^{2} .
$$

We explain some notations about relations between given bounded operators $T_{1}, T_{2}$. Suppose there is an intertwining bounded operator $X$ such that $X T_{1}=$ $T_{2} X$, then we denote by $T_{1} \stackrel{d}{\prec} T_{2}, T_{1} \stackrel{i}{\prec} T_{2}, T_{1} \prec T_{2}, T_{1} \approx T_{2}$, and $T_{1} \cong T_{2}$,

Received by the editors February 19, 1988 and, in revised form, August 25, 1988.

1980 Mathematics Subject Classification (1985 Revision). Primary 47A65, 47A45, 47A70.

Key words and phrases. Curvature of holomorphic vector bundle, Szegö kernel, contraction, quasi-similar, similar, Hardy class, corona theorem, eigenvector, canonical model theory. 
$X$ with dense range, $X$ injective, quasi-affinity (that is, $X$ is injective and has dense range), invertible, and unitary, respectively. Moreover we write $T_{1} \sim T_{2}$ and say that $T_{1}$ and $T_{2}$ are quasi-similar, if $T_{1} \prec T_{2}$ and $T_{2} \prec T_{1}$. In [4], Cowen-Douglas defined the class $B_{n}(\Omega)$ consisting of bounded operator $T$ satisfying

(a) $\Omega \subset \sigma(T)$,

(b) range $(T-w)=H$ for each $w$ in $\Omega$,

(c) $\bigvee_{w \in \Omega} \operatorname{ker}(T-w)=H$,

(d) $\operatorname{dim} \operatorname{ker}(T-w)=n$ for $w$ in $\Omega$.

Now we introduce the class $B_{n}^{h}(\Omega)$ as

Definition. $T$ belongs to $B_{n}^{h}(\Omega)$ if there is a holomorphic curve $\gamma: \Omega \rightarrow$ $g_{r}(n, H)$ such that $\gamma(w) \subset \operatorname{ker}(T-w)$, and $\bigvee_{w \in \Omega} \gamma(w)=H$. It is known that $B_{n}(\Omega) \subset B_{n}^{h}(\Omega)$. If $T$ is in $B_{n}^{h}(\Omega)$, then the bundle is well defined by the curve $\gamma(w)$. We denote it and its curvature by $E_{T}$ and $\mathscr{K}_{T}$.

The purpose of this paper is to estimate $\mathscr{K}_{T}$ of $T$ in $B_{n}^{h}(\Omega)$ and to research what kind of operator is similar or quasi-similar to the shifts.

Now we show some examples. Let $\left\{e_{n}\right\}_{n=0}^{\infty}$ be a C.O.N.B. of $H$ and $A$ a weighted shift with positive weight $\left\{a_{n}\right\}_{n=1}^{\infty}$, that is $A e_{n}=a_{n+1} e_{n+1}$. Set $b_{n}=a_{1} \cdots a_{n}$ and $r_{1}(A)=\lim _{n \rightarrow \infty}\left(\inf _{k} b_{k+n} / b_{k}\right)^{1 / n}$. Then we have $A^{*} \in$ $B_{1}\left(\left\{w:|w|<r_{1}(A)\right\}\right)$, (see [13 or 12]). Especially, the adjoint of unilateral shift $S$ corresponding to $a_{n}=1$ for all $n$ and the adjoint of the Bergman shift $B$ corresponding to $a_{n}=\sqrt{n /(n+1)}$ for all $n$ are both in $B_{1}(D)$, where $D$ is the open unit disk. And $\mathscr{K}_{S^{*}}(w)=-1 /\left(1-|w|^{2}\right)^{2}$ and $\mathscr{K}_{B^{*}}(w)=-2 /\left(1-|w|^{2}\right)^{2}$.

In $[17,18]$ we studied a contraction $T$ with $I-T^{*} T$ in the trace class, and showed that $S_{n}^{*} \prec T^{*}$ if and only if $T$ is in $C_{10}$ (that is, $T^{n} x \nrightarrow 0$, $T^{* n} x \rightarrow 0$ as $n \rightarrow \infty$ for every $x \neq 0$ ) [17], and that these are equivalent with $T^{*} \in B_{n}^{h}(D)$ [18]. We should notice that $B_{n}^{h}(\Omega) \subset B_{n}^{h}(\Delta)$ for $\Delta \subset \Omega$ (cf. p. 193 of [4]).

\section{Curvatures}

It was shown that the curvature of a vector bundle generated by a holomorphic curve was nonpositive, and if $T$ is in $B_{1}(\Omega)$, then

$$
\mathscr{K}_{T}(w)^{-1}=-\operatorname{trace} N_{w}^{*} N_{w},
$$

where $N_{w}=\left.(T-w)\right|_{\operatorname{ker}(T-w)^{2}}$ [4]. Let $\Omega$ be a finitely connected Jordan region and $\operatorname{cl} \Omega$ (closure of $\Omega$ ) is a spectral set for $T$, that is $\sigma(T) \subset \operatorname{cl} \Omega$ and $\|f(T)\| \leq\|f\|_{\infty}$ for every rational function $f$ with no poles in $\mathrm{cl} \Omega$. Then the curvature of $T$ in $B_{1}(\Omega)$ was estimated by Misra [9] as

$$
\mathscr{K}_{T}(w) \leq-\widehat{K}_{\Omega}(w, \bar{w})^{2},
$$

where $\widehat{K}_{\Omega}$ is the Szegö kernel of $\Omega$. His proof is based on (2.1). In this section we will extend (2.2) to the case of the $B_{n}^{h}(\Omega)$ by virtue of the canonical model 
theory of contraction due to Sz.-Nagy and Foias [14]; let $T$ be a contraction on $H$ in $C_{.0}$, that is $T^{* n} x \rightarrow 0$ for $x$ in $H$. Then there is the characteristic function $\theta(z)$, which is a $B\left(F_{1}, F_{2}\right)$-valued holomorphic contractive function defined on $D$ and $\theta(z)$ is isometric from $F_{1}$ to $F_{2}$ a.e. on the unit circle, where $F_{1}$ and $F_{2}$ are the subspaces of $H$ called defect spaces of $T$. And then $T$ on $H$ is unitarily equivalent to $S(\theta)$ on $H(\theta)$ given as the following:

$$
H(\theta)=H^{2}\left(F_{2}\right) \ominus \theta H^{2}\left(F_{1}\right), \quad S(\theta)^{*}=\left.M_{z}^{*}\right|_{H(\theta)},
$$

where $M_{z}$ is the multiplication by $z$ on $H^{2}\left(F_{2}\right)$, which is the Hardy class of $F_{2}$-valued holomorphic functions on $D$. We remark that $S_{n}:=S \oplus \cdots \oplus S \cong M_{z}$ on $H^{2}\left(\mathbf{C}_{n}\right)$.

Theorem 2.1. Let $\gamma: \Omega \rightarrow g_{r}(n, H)$ be a holomorphic curve such that $\Omega \subset$ $D, \Omega$ is open, $\bigvee_{w \in \Omega} \gamma(w)=H$. Suppose there is a contraction $T$ such that $\gamma(w) \subset \operatorname{ker}\left(T^{*}-w\right)$ for $w \in \Omega$. Then $\mathscr{K}_{\gamma}(w) \quad\left(=\mathscr{K}_{T^{*}}(w)\right) \leq-I_{n} /\left(1-|w|^{2}\right)^{2}$ for $w$ in $\Omega$.

Proof. Since $T^{* k} \gamma(w)=w^{k} \gamma(w) \rightarrow 0(k \rightarrow \infty),\left\|T^{*}\right\| \leq 1$ implies $T \in C_{._{0}}$. So we may consider $S(\theta)$ of (2.3) instead of $T$. For any $w_{0} \in \Omega$, there is a nbhd $\Delta$ of $w_{0}$ and a frame $\gamma_{1 w}, \ldots, \gamma_{n w}$ for $\gamma_{w}$ on $\Delta$. Then, since $M_{z}^{*} \gamma_{i w}=w \gamma_{i w}$, we can represent $\gamma_{i w}$ as the function in $H(\theta)$ :

$$
\gamma_{i w}(z)=\frac{\gamma_{i w}(0)}{1-w z} \quad \text { for } z \in D
$$

Thus we have

$$
\gamma_{i w}(0) \perp \theta(\bar{w}) F_{2}
$$

and

$$
\begin{aligned}
\left(\gamma_{j w}, \gamma_{i w}\right)_{H(\theta)} & =\frac{1}{2 \pi} \int_{\partial D}\left(\gamma_{j w}(z), \gamma_{i w}(z)\right)_{F_{2}}|d z| \\
& =\frac{1}{1-|w|^{2}}\left(\gamma_{j w}(0), \gamma_{i w}(0)\right)_{F_{2}},
\end{aligned}
$$

which implies $\gamma_{i w}(0), \ldots, \gamma_{n w}(0)$ are linearly independent. Hence, if we set $\gamma_{w}^{0}=\bigvee\left\{\gamma_{i w}(0): i=1, \ldots, n\right\}$ for each $w \in \Delta$, then $\gamma^{0}: \Delta \rightarrow g_{r}\left(n, F_{2}\right)$ is a holomorphic curve. From (1.1) and (2.6), it follows that

$$
\mathscr{K}_{\gamma}(w)=-\frac{I_{n}}{\left(1-|w|^{2}\right)^{2}}+\mathscr{K}_{\gamma^{0}}(w) \text { for } w \text { in } \Delta .
$$

Since $\mathscr{K}_{\gamma^{0}}(w) \leq 0$, we can conclude the proof.

Proposition 2.2. If $T$ is a contraction in $B_{n}^{h}(D)$ and $\mathscr{K}_{T}(w)=I_{n} /\left(1-|w|^{2}\right)^{2}$ on an open set $\Delta \subset D$, then $T \cong S_{n}^{*}$.

Proof. Since $\mathscr{K}_{T}(w)=\mathscr{K}_{S_{n}^{*}}(w)$ for $w$ in $\Delta$, from Proposition 3.3 of [4], there is a holomorphic isometric bundle map $U(w)$ satisfying $U(w) \operatorname{ker}(T-w)=$ 
$\operatorname{ker}\left(S_{n}^{*}-w\right)$ for $w$ in $\Delta$. Since $T$ is in $B_{n}^{h}(\Delta)$, by the rigidity theorem (cf. p. 202 of [4]), there is a unitary $U$ on $H$ such that $U \operatorname{ker}(T-w)=\operatorname{ker}\left(S_{n}^{*}-w\right)$ and hence $U T=S_{n}^{*} U$. Thus the proof is complete.

Let $\Omega_{1}, \Omega_{2}$ be connected open sets, $\gamma: \Omega_{2} \rightarrow g_{r}(n, H)$ a holomorphic curve, and $\phi$ an injective holomorphic mapping from $\Omega_{1}$ to $\Omega_{2}$. Then by the chain rule and (1.1) we have

$$
\mathscr{K}_{\gamma \circ \phi}(w)=\left|\phi^{\prime}(w)\right|^{2} \mathscr{K}_{\gamma}(\phi(w)) \text { for } w \text { in } \Omega_{1} .
$$

Proposition 2.3. If $T$ is a bounded operator in $B_{n}(\Omega)$, where $\Omega$ is an open connected set, then

$$
\mathscr{K}_{T}(w) \leq-\frac{I_{n}}{\left(\|T\|^{2}-|w|^{2}\right)^{2}} \quad \text { for } w \in \Omega .
$$

Proof. From (2.8) $\mathscr{K}_{T /\|T\|}(w /\|T\|)=\|T\|^{2} \mathscr{K}_{T}(w)$ follows. Since $\Omega /\|T\| \subset D$, Theorem 2.1 implies the above inequality.

Theorem 2.5. Let $\Omega$ be a p-ply connected Jordan region, and $T \in B_{n}^{h}(\Delta)$ for some $\Delta \subset \Omega$. Suppose $\mathrm{cl} \Omega$ is a spectral set of $T$. Then we have

$$
\mathscr{K}_{t}(w) \leq-\widehat{K}_{\Omega}(w, \bar{w})^{2} I_{n} \quad \text { for } w \in \Delta .
$$

Proof. For each $w_{0}$ in $\Delta$ there is a holomorphic function $F$ from $\Omega$ to a $p$-sheeted disc such that $F\left(w_{0}\right)=0, F^{\prime}\left(w_{0}\right) \neq 0$, and $F$ is continuous on $\operatorname{cl} \Omega$ (cf. $[7,2])$. From Mergerlyan's theorem there is a sequence of rational functions with no poles in $\mathrm{cl} \Omega$ which uniformly converges to $F$ on $\mathrm{cl} \Omega$. We denote it by $\left\{R_{n}\right\}$. Then Riesz functional $R_{n}(T)$ is well defined and $\left\{R_{n}(T)\right\}$ converges uniformly. We represent its limit by $F(T)$. Then for a holomorphic curve $\gamma(w) \subset \operatorname{ker}(T-w)$ on $\Delta,\|F(T)\| \leq\|F\|=1$, and $\{F(T)-F(w)\} \gamma(w)=0$ follows, because $\left\{R_{n}(T)-R_{n}(w)\right\} \gamma(w)=0$. From $F^{\prime}\left(w_{0}\right) \neq 0$ we can take neighbourhoods $\Omega_{1}$ of $w_{0}$ and $\Omega_{2}$ of 0 such that $\left.F\right|_{\Omega_{1}}: \Omega_{1} \rightarrow \Omega_{2}$ is bijective. Let $\phi$ be the inverse of $F \mid \Omega_{1}$. Then we have $\{F(T)-z\} \gamma(\phi(z))=0$ for $z$ in $\Omega_{2}$. Since

$$
\bigvee\left\{\gamma(\phi(z)): z \in \Omega_{2}\right\}=\bigvee\left\{\gamma(w): w \in \Omega_{1}\right\}=\bigvee\{\gamma(w): w \in \Omega\}=H
$$

follows from p. 194 of [4], a contraction $F(T)$ and curve $\gamma \circ \phi$ satisfy the conditions of Theorem 2.1. Thus at the origin $\mathscr{K}_{\gamma \circ \phi}(0) \leq-I_{n}$, from which, using (2.8), we get

$$
\mathscr{K}_{\gamma}\left(w_{0}\right) \leq-\left|F^{\prime}\left(w_{0}\right)\right|^{2} I_{n}=-\widehat{K}_{\Omega}\left(w_{0}, \bar{w}_{0}\right)^{2} I_{n},
$$

because the second equality follows from p. 118 of [2]. Consequently we can conclude the proof.

At the end of this section we consider the question proposed on p. 329 of [5], that is, if $T_{1}$ and $T_{2}$ are contractions in $B_{1}(D)$ such that $\mathscr{K}_{T_{1}} \leq \mathscr{K}_{T_{2}}$, then does there exist a bounded operator $X$ such that $X T_{1}=T_{2} X$ ? Corollary 2.2 shows $\mathscr{K}_{T} \leq \mathscr{K}_{S^{*}}$ for any contraction $T$ in $B_{1}(D)$, and the existence of $X$ 
with dense range satisfying $X T=S^{*} X$ is well known (cf. [16], or see the proof of Proposition 3.6). Hence the question is true in the case of $T_{2}=S^{*}$. In [10] Misra showed that a contraction $T$ in $B_{1}(D)$ is unitarily equivalent to $\phi(T)$ for every Möbius transformation $\phi$ of $D$ if and only if $\mathscr{K}_{T}(w)=-\alpha /\left(1-|w|^{2}\right)^{2}$, where $\alpha$ is a constant and $\alpha \geq 1$.

Proposition 2.6. Let $T_{1}, T_{2}$ be contractions in $B_{1}(D)$ with curvature $\mathscr{K}_{T_{i}}(w)=$ $-\alpha_{i} /\left(1-|w|^{2}\right)^{2} \quad\left(\alpha_{i} \geq 1\right)$. Then next conditions are equivalent: (i) $\mathscr{K}_{T_{2}} \leq \mathscr{K}_{T_{1}}$, (ii) there is a bounded operator $X$ such that $X T_{2}=T_{!} X$, and (iii) $T_{2} \prec T_{1}$. Proof. Let $A_{i}$ be the weighted shift with weight $a_{n i}=\sqrt{n /\left(\alpha_{i}+n-1\right)}$ for $i=1,2$. Then we have $r_{1}\left(A_{i}\right)=1$ and hence $A_{i}^{*} \in B_{1}(D)$. Since the square of the norm of a holomorphic eigenvector of $A_{i}^{*}-w$ is $\left(1-|w|^{2}\right)^{\alpha_{i}}$, $\mathscr{K}_{A_{i}^{*}}(w)=\mathscr{K}_{T_{i}}(w)$, and hence $A_{i}^{*} \cong T_{i}$ (see [5]). Thus we may identify $A_{i}^{*}$ with $T_{i}$. Assume (i). Then diagonal quasi-affinity $Y$ defined by $Y e_{n}=$ $\left\{\left(a_{12} \cdots a_{n 2}\right) /\left(a_{11} \cdots a_{n 1}\right)\right\} e_{n}$ satisfies $Y A_{1}=A_{2} Y$ and hence $Y^{*} T_{2}=$ $T_{1} Y^{*}$, which implies (iii). Assume (ii). Since $X^{*} A_{1}=A_{2} X^{*}$, setting $b_{m n}=$ $\left(X^{*} e_{n}, e_{m}\right)$, we obtain

$$
b_{m n+1} a_{n+11}=\left\{\begin{array}{l}
0 \quad(m=0), \\
b_{m-1 n} a_{m 2}
\end{array} \quad(m \geq 1) .\right.
$$

Since there is a nonvanishing $b_{i j}(i \geq j)$, boundedness of $X$ implies that $\prod_{k=1}^{\infty} a_{i+k 2} / a_{j+k 1}$ is bounded. To show (i), suppose $\alpha_{1}>\alpha_{2}$, then each term of the infinite product is larger than 1 . Hence

$$
\sum_{k=1}^{\infty}\left(\left(\frac{\alpha_{1}+j+k-1}{j+k} / \frac{\alpha_{2}+i+k-1}{i+k}\right)-1\right)
$$

must converge, however this is impossible. Consequently (i) follows. (iii) obviously implies (ii), and the proof is complete.

We can apply the previous result to show that $S \prec B$, where $B$ is the Bergman shift, but there is not a bounded operator $X$ such that $X B=S X$, though it is possible to get them by another simple method.

\section{EXACT SEQUENCE AND INTERTWINING OPERATORS}

In this section we give the conditions for a contraction $T$ to be $T \prec S_{n}$ or $T \approx S_{n}$. At the beginning we will refer to a result about exact sequence of Hardy classes and use it to show that if $T \prec S_{n}$, then $T^{*} \in B_{n}(D)$. A $B\left(F_{1}, F_{2}\right)$ valued holomorphic function $\Gamma(z)$ on $D$ is called bounded if $\sup _{z \in D}\|\Gamma(z)\|<$ $\infty$. In this case a bounded operator $\Gamma$ from $H^{2}\left(F_{1}\right)$ to $H^{2}\left(F_{2}\right)$ is determined by $(\Gamma f)(z)=\Gamma(z) f(z)$.

Theorem 3.1. Let $\Gamma_{1}, \Gamma_{2}$ be operator-valued bounded holomorphic functions on $D$, and suppose

$$
H^{2}\left(F_{1}\right) \stackrel{\Gamma_{1}}{\rightarrow} H^{2}\left(F_{2}\right) \stackrel{\Gamma_{2}}{\rightarrow} H^{2}\left(\mathbf{C}_{n}\right)
$$


is exact and $\Gamma_{2}$ has the dense range. Then the next sequence is exact for every $z$ in $D$ :

$$
F_{1} \stackrel{\Gamma_{1}(z)}{\rightarrow} F_{2} \stackrel{\Gamma_{2}(z)}{\rightarrow} \mathbf{C}_{n} \rightarrow 0
$$

Proof. Since $\Gamma_{2}(z) \Gamma_{1}(z)=0$, we have only to show $\operatorname{ker} \Gamma_{2}(z) \subset \Gamma_{1}(z) F$. Since $\Gamma_{2}$ has the dense range, from the Cauchy integral formula, the range of $\Gamma_{2}(z)$ is dense and hence coincident with $C_{n}$. Thus $\Gamma_{2}^{\sim}(z):=\Gamma_{2}(\bar{z})^{*}$ is injective with closed range. Fix an arbitrary $z_{0}$ in $D$. There is an isometry $V$ from $C_{n}$ to $F_{2}$ such that $\operatorname{det} V^{*} \Gamma_{2}^{\sim}\left(z_{0}\right) \neq 0$. Then $\Omega:=\left\{z \in D: \operatorname{det} V^{*} \Gamma_{2}^{\sim}(z)=0\right\}$ is a set of isolated points. In the same way as Theorem 1 of [17] or p. 94 of [8] we can obtain a $B\left(F, F_{2}\right)$-valued bounded holomorphic function $\Phi(z)$ defined on $D$ such that $\Gamma_{2}^{\sim}(z) C_{n} \oplus \Phi(\bar{z}) F=F_{2}$ for $z \in D \backslash \Omega$, where $F$ is an auxiliary Hilbert space. This implies $\operatorname{ker} \Gamma_{2}(\bar{z})=\Phi(\bar{z}) F$ for $z \in D \backslash \Omega$ and hence $\Gamma_{2} \Phi=0$. Thus we have $\Phi H^{2}(F) \subset \operatorname{ker} \Gamma_{2}=\Gamma_{1} H^{2}\left(F_{1}\right)$. Taking $F$ valued constant functions we get $\Phi(z) F \subset \Gamma_{1}(z) F_{1}$ for $z \in D$. Thus we have $\operatorname{ker} \Gamma_{2}\left(\bar{z}_{0}\right)=\Phi\left(\bar{z}_{0}\right) F \subset \Gamma_{1}\left(\bar{z}_{0}\right) F_{1}$. The proof is complete.

Remark. The converse assertion of the theorem is false. In fact, set

$$
\Gamma_{1}(z)=\left(\begin{array}{c}
\exp \frac{z+1}{z-1} \\
0
\end{array}\right), \quad \Gamma_{2}(z)=(0,1),
$$

then

$$
C_{1} \stackrel{\Gamma_{1}(z)}{\rightarrow} C_{2} \stackrel{\Gamma_{2}(z)}{\rightarrow} C_{1} \rightarrow 0
$$

is exact for each $z$, but

$$
\Gamma_{1} H^{2}\left(C_{1}\right)=\exp \frac{z+1}{z-1} H^{2}\left(C_{1}\right) \oplus 0 \varsubsetneqq H^{2}\left(C_{1}\right) \oplus 0=\operatorname{ker} \Gamma_{2} .
$$

Corollary 3.2 (K. Takahashi [16]). Let $T$ be a contraction with $T \prec S_{n}$, then $T^{*} \in B_{n}(D)$.

Proof. Since $T$ is in class $C_{._{0}}$, we may identify $S(\theta)$ given by (2.3) with $T$. Let $X$ be a quasi-affinity such that $X S(\theta)=S_{n} X$. Then, from the lifting theorem (see [14]) there is a $B\left(F_{2}, C_{n}\right)$-valued bounded holomorphic function $\Gamma(z)$ defined on $D$ such that $\Gamma \theta=0$ and $X h=\Gamma h$ for $h$ in $H(\theta)$. That $X$ is a quasi-affinity implies that

$$
H^{2}\left(F_{1}\right) \stackrel{\theta}{\rightarrow} H^{2}\left(F_{2}\right) \stackrel{\Gamma}{\rightarrow} H^{2}\left(C_{n}\right)
$$

is exact, and that $\Gamma$ has the dense range. Thus from the theorem we get $\theta(w) F_{1}$ is closed and $\operatorname{dim}\left\{F_{2} \ominus \theta(w) F_{1}\right\}=n$ for $w$ in $D$. The next equivalent conditions:

(1) $\theta(w) F_{1}$ is closed in $F_{2}$,

(2) $\frac{z-w}{1-\bar{w} z} H^{2}\left(F_{2}\right) \oplus \frac{\theta(w) F_{1}}{1-\bar{w} z}$ is closed in $H^{2}\left(F_{2}\right)$,

(3) $\frac{z-w}{1-\bar{w} z} H^{2}\left(F_{2}\right)+\theta H^{2}\left(F_{1}\right)$ is closed in $H^{2}\left(F_{2}\right)$,

(4) $P_{H(\theta)} \frac{z-w}{1-\bar{w} z} H(\theta)$ is closed in $H(\theta)$,

(5) $(S(\theta)-w)(I-\bar{w} S(\theta))^{-1} H(\theta)$ is closed in $H(\theta)$, 
show that the range of $(S(\theta)-w)^{*}$ is closed for $w$ in $D$. Similarly we have $\operatorname{dim} \operatorname{ker}(S(\theta)-w)^{*}=n$, hence the proof is complete.

Remark. The latter half in the above proof is trivial if we notice that $\theta$ is the characteristic function of $S(\theta)$ [14]. But we showed it directly.

Theorem 3.3. Let $T$ be a contraction. Then $T \prec S_{n}$ if and only if $T^{*} \in B_{n}^{h}(D)$ and there is a frame $\left\{\gamma_{1} w, \ldots, \gamma_{n w}\right\}$ for $\operatorname{ker}\left(T^{*}-w\right)$ on $D$ such that

$$
\sup _{w \in D}\left(1-|w|^{2}\right)\left\|\gamma_{i w}\right\|^{2}<\infty \text { for each } i \text {. }
$$

Proof. Let $\left\{e_{1}, \ldots, e_{n}\right\}$ be the O.N.B. of $\mathbf{C}_{n}$. Then eigenvectors of $\left(S_{n}^{*}-w\right)$ are $e_{1} /(1-w z), \ldots, e_{n} /(1-w z)$. If $X$ is the quasi-affinity such that $X T=$ $S_{n} X$, then $\gamma_{i w}=X^{*} e_{i} /(1-w z)$ satisfies the norm condition. The rest of "only if" part is clear. In order to show "if" part, we consider $S(\theta)$ instead of $T$. Then $\gamma_{i w}$ is given by (2.4). By the norm condition and (2.6), $\left\|\gamma_{i w}(0)\right\|$ is uniformly bounded for $w$ in $D$. For each $z$ in $D$, we determine the operator $\Gamma(z): F_{2} \rightarrow \mathbf{C}_{n}$ by

$$
\Gamma(z) y=\sum_{i=1}^{n}\left(y, \gamma_{i \bar{z}}(0) e_{i} .\right.
$$

Then from (2.5) we have $\Gamma(z) \theta(z)=0$, and clearly $\sup _{z \in D}\|\Gamma(z)\|<\infty$. Let us determine the bounded operator $X: H(\theta) \rightarrow H^{2}\left(\mathbf{C}_{n}\right)$ by $X h=\Gamma h$ for $h$ in $H(\theta)$. Then it clearly follows that $X S(\theta)=S_{n} X$. For any $i, k$, and any $\zeta$, $w$ in $D$, since $z$ is the variable of a function, we have

$$
\begin{aligned}
& \left(X^{*} \frac{e_{i}}{1-w z}, \frac{\gamma_{k \zeta}(0)}{1-\zeta z}\right)_{H(\theta)}=\left(\frac{e_{i}}{1-w z}, \sum_{j} \frac{\left(\gamma_{k \zeta}(0), \gamma_{j \bar{z}}(0)\right) e_{j}}{1-\zeta z}\right)_{H^{2}\left(C_{n}\right)} \\
& \quad=\left(\frac{\gamma_{i \bar{z}}(0)}{1-w z}, \frac{\gamma_{k \zeta}(0)}{1-\zeta z}\right)_{L^{2}\left(F_{2}\right)}=\left(P_{H^{2}\left(F_{2}\right)} \frac{\gamma_{i \bar{z}}(0)}{1-w z}, \frac{\gamma_{k \zeta}(0)}{1-\zeta z}\right)_{H^{2}\left(F_{2}\right)} \\
& \quad=\left(\frac{\gamma_{i w}(0)}{1-w z}, \frac{\gamma_{k \zeta}(0)}{1-\zeta z}\right)_{H(\theta)}=\left(\gamma_{i w}, \gamma_{k \zeta}\right)_{H(\theta)},
\end{aligned}
$$

which shows that $X^{*} e_{i} /(1-w z)=\gamma_{i w}$, because $\bigvee_{k \zeta} \gamma_{k \zeta}=H(\theta)$, and hence that $X^{*}$ has the dense range. Thus $X$ is injective. Since the rank of $\Gamma(z)$ is $n,\left.S_{n}\right|_{\mathrm{cl} X H(\theta)}=\left.S_{n}\right|_{\mathrm{cl} \Gamma H^{2}\left(F_{2}\right)}$ is unitarily equivalent to $S_{n}$. To accomplish the proof, it suffices to take $P X$ to be the intertwining quasi-affinity, where $P$ is the projection from $H^{2}\left(C_{n}\right)$ to $\operatorname{cl} X H(\theta)$. The proof is complete.

Suppose $T$ be a completely nonunitary (c.n.u.) contraction. In [1], Alexander called vectors $h_{1}, \ldots, h_{n}$ analytically independent under $T$ if a relation $\phi_{1}(T) h_{1}+\cdots+\phi_{n}(T) h_{n}=0$ with $\phi_{i} \in H^{\infty}$ implies $\phi_{1}=\cdots=\phi_{n}=0$, and showed that $S_{n} \prec T$ if and only if $T$ has $n$ cyclic vectors which are analytically independent under $T$. We remark that a contraction $T$ with the adjoint in $B_{n}^{h}(D)$ satisfies $T^{* n} \rightarrow 0$ so that $T$ is c.n.u. 
Corollary 3.4. Let $T$ be a contraction. Then $T \sim S_{n}$ if and only if $T$ has $n$ cyclic vectors, $T^{*} \in B_{n}^{h}(D)$ and there is a frame $\left\{\gamma_{1^{w}}, \ldots, \gamma_{n w}\right\}$ for $\operatorname{ker}\left(T^{*}-w\right)$ on $D$ such that

$$
\sup _{w \in D}\left(1-|w|^{2}\right)\left\|\gamma_{i w}\right\|^{2}<\infty \quad \text { for each } i \text {. }
$$

Proof. We have only to show "if" part. From above theorem $T \prec S_{n}$ follows. Let $X$ be a quasi-affinity satisfying $X T=S_{n} X$, and $h_{1}, \ldots, h_{n}$ cyclic vectors for $T$. Then $X h_{1}, \ldots, X h_{n}$ are cyclic vectors for $S_{n}$. It is trivial to show that for each $z$ in $D\left(X h_{1}\right)(z), \ldots,\left(X h_{n}\right)(z)$ span $C_{n}$ and hence $\operatorname{det}\left(\left(X h_{1}\right)(z), \ldots,\left(X h_{n}\right)(z)\right) \neq 0$. Thus, from [1], $X h_{1}, \ldots, X h_{n}$ are analytically independent under $S_{n}$. Since $X \phi_{i}(T) h_{i}=\phi_{i}\left(S_{n}\right)\left(X h_{i}\right), h_{1}, \ldots, h_{n}$ are analytically independent under $T$. Thus we obtain $S_{n} \prec T$ and hence $S_{n} \sim T$.

In [20], P. Y. Wu gave a necessary and sufficient condition for the characteristic function of $T$ to be $T \sim S_{n}$. That $S_{n}^{*}$ has a cyclic vector was shown by D. Sarason. Now we can extend it as follows:

Theorem 3.5. If $\Omega$ is a connected open set and $T^{*} \in B_{n}^{h}(\Omega)$, then $T^{*}$ has a cyclic vector. Especially if $T$ is a contraction with $T^{*} \in B_{n}^{h}(D)$, then $S \prec T^{*}$. Proof. Fix an arbitrary $w_{0}$ in $\Omega$, then there is a nbhd $\Delta$ of $w_{0}$, and a frame $\gamma_{1 w}, \ldots, \gamma_{n w}$ for $\operatorname{ker}\left(T^{*}-w\right)$ on $\Delta$. Since $B_{n}^{h}(\Omega) \subset B_{n}^{h}(\Delta)$,

$$
\bigvee\left\{\gamma_{i w}: 1 \leq i \leq n, w \in \Delta\right\}=H
$$

follows. By the Taylor expansion we have $\bigvee\left\{\gamma_{i}^{(k)}: 1 \leq i \leq n, 1 \leq k<\infty\right\}=H$, where $\gamma_{i}^{(k)}=\left(d^{k} \gamma_{i w} / d w^{k}\right)_{w=w_{0}} \in H$. From $\left(T^{*}-w\right) \gamma_{i w}=0$, it follows that $\left(T^{*}-w_{0}\right) \gamma_{i}^{(k)}=k \gamma_{i}^{(k-1)}$. Setting $a_{k}=1 / k$ ! , clearly $\sum_{k=0}^{\infty}\left\|\gamma_{i}^{(k)}\right\| a_{k} / k !<\infty$. In case of $n=1, x=\sum_{k=0}^{\infty} \gamma_{1}^{(k)} a_{k} / k$ ! is a cyclic vector. In fact,

$$
\left(T^{*}-w_{0}\right)^{m} x=\sum_{k=0}^{\infty} \frac{\gamma_{1}^{(k)}}{k !} a_{m+k}
$$

implies that

$$
\left\|\frac{\left(T^{*}-w_{0}\right)^{m}}{a_{m}} x-\gamma_{1}^{(0)}\right\| \leq \frac{a_{m+1}}{a_{m}} \sum_{k=1}^{\infty} \frac{\left\|\gamma_{1}^{(k)}\right\|}{k !} \frac{a_{m+k}}{a_{m+1}} \leq \frac{a_{m+1}}{a_{m}}\left(\sum_{k=1}^{\infty} \frac{\left\|\gamma_{1}^{(k)}\right\|}{k !} \frac{a_{k}}{a_{1}}\right) \rightarrow 0
$$

as $m \rightarrow \infty$. Thus $\gamma_{1}^{(0)} \in \bigvee_{m=0}^{\infty}\left(T^{*}-w_{0}\right)^{m} x$. From

$$
\begin{aligned}
& \left\|\frac{1}{a_{m}}\left(\left(T^{*}-w_{0}\right)^{m-1} x-a_{m-1} \gamma_{1}^{(0)}\right)-\gamma_{1}^{(1)}\right\| \\
& \quad \leq \frac{a_{m+1}}{a_{m}} \sum_{k=2}^{\infty} \frac{\left\|\gamma_{1}^{(k)}\right\|}{k !} \frac{a_{k}}{a_{2}} \rightarrow 0 \quad(m \rightarrow \infty),
\end{aligned}
$$

we have $\gamma_{1}^{(1)} \in \bigvee_{m=0}^{\infty}\left(T^{*}-w_{0}\right)^{m} x$. Similarly we get $\gamma_{1}^{(k)} \in \bigvee_{m=0}^{\infty}\left(T^{*}-w_{0}\right)^{m} x$, consequently $\bigvee_{m=0}^{\infty}\left(T^{*}-w_{0}\right)^{m} x=H$, and hence $\bigvee_{m=0}^{\infty} T^{* m} x=H$. In case of 
$n>1$

$$
\begin{aligned}
x= & \gamma_{1}^{(0)} a_{0}+\frac{\gamma_{2}^{(1)}}{1 !} a_{1}+\frac{\gamma_{3}^{(2)}}{2 !} a_{2}+\cdots+\frac{\gamma_{n}^{(n-1)}}{(n-1) !} a_{n-1} \\
& +\frac{\gamma_{1}^{(n)}}{n !} a_{n}+\frac{\gamma_{2}^{(n+1)}}{(n+1) !} a_{n+1}+\cdots
\end{aligned}
$$

is a cyclic vector for $T^{*}$. To show the rest, suppose $\phi\left(T^{*}\right) x=0$ for $\phi \in H^{\infty}$. Since $\phi\left(T^{*}\right) T^{* m} x=T^{* m} \phi\left(T^{*}\right) x=0$, we have $\phi\left(T^{*}\right)=0$. From $T^{*} \gamma_{i w}=$ $w \gamma_{i w}$, it follows that $\phi\left(T^{*}\right) \gamma_{i w}=\phi(w) \gamma_{i w}$ for every $w$ in $D$ and hence $\phi(w)=$ 0 , which implies that $x$ is analytically independent under $T^{*}$. Consequently we get $S \prec T^{*}$.

Proposition 3.6. If $T$ is a contraction and $T \prec S_{n}$, then there is an invariant subspace $L$ for $T$ such that $\left.T\right|_{L} \sim S_{n}$.

Proof. Let us consider $S(\theta)$ instead of $T$. Then the eigenvector $\gamma_{i 0}$ of $T^{*}$ is given by (2.4). Since it is constant vector valued, we can determine a bounded operator $Y$ from $H^{2}\left(\mathbf{C}_{n}\right)=H^{2}\left(\mathbf{C}_{1}\right) \oplus \cdots \oplus H^{2}\left(\mathbf{C}_{1}\right)$ to $H(\theta)$ by

$$
Y\left(h_{1} \oplus \cdots \oplus h_{n}\right)=P_{H(\theta)}\left(h_{1} \gamma_{10}+\cdots+h_{n} \gamma_{n 0}\right) \text {. }
$$

Suppose $Y\left(h_{1} \oplus \cdots \oplus h_{n}\right)=0$. Then $\sum h_{i} \gamma_{i 0} \in \theta H^{2}\left(F_{1}\right)$ so that there is $f$ in $H^{2}\left(F_{1}\right)$ such that $\sum h_{i} \gamma_{i 0}=\theta f$. By (2.5) and linear independence of $\gamma_{10}(0), \ldots, \gamma_{n 0}(0)$, we have $h_{i}(0)=0$ and $f(0)=0$. Since

$$
\sum h_{i}^{\prime}(0) \gamma_{i 0}(0)=\theta^{\prime}(0) f(0)+\theta(0) f^{\prime}(0)=\theta(0) f^{\prime}(0) \text {, }
$$

we have $h_{i}^{\prime}(0)=0$ and $f^{\prime}(0)=0$ too. Thus to show $h_{i}=0$ it suffices to continue this process. Set $L=\operatorname{cl} Y H^{2}\left(\mathbf{C}_{n}\right)$. Then $T L \subset L$ and $\left.S_{n} \prec T\right|_{L}$. Let $X$ be a quasi-affinity satisfying $X T=S_{n} X$. Then $X Y$ is injective and commutes with $S_{n}$. From the characterizations of invariant subspaces for $S_{n}$, it follows that $\left.S_{n}\right|_{\mathrm{cl} X L}=\left.S_{n}\right|_{\mathrm{cl} X Y H^{2}\left(\mathbf{C}_{n}\right)} \cong S_{n}$, and hence $\left.T\right|_{L} \prec S_{n}$. Thus we have $\left.T\right|_{L} \sim S_{n}$ and the proof is complete.

Next we will give the conditions for contractions to be similar to $S_{n}$ by using the Rosenblum's infinite corona theorem [11]. Suppose

$$
\sup _{z \in D} \sum_{j=1}^{n} \sum_{i=1}^{\infty}\left|h_{i j}(z)\right|^{2}<\infty, \quad \text { where } h_{i j} \in H^{\infty} \text {. }
$$

Then a $B\left(\mathbf{C}_{n}, l^{2}\right)$-valued holomorphic function $A(z)=\left(h_{i j}(z)\right)$ is bounded on $D$. Under this setting we have

Proposition 3.7. There is a $B\left(l^{2}, \mathbf{C}_{n}\right)$-valued bounded holomorphic function $B(z)$ such that $B(z) A(z)=I$ for $z$ in $D$, if and only if there is a positive constant $\delta$ such that $\|A(z) x\| \geq \delta\|x\|$ for every $x$ in $\mathbf{C}_{n}$ and every $z$ in $D$.

Proof. Suppose $\|A(z) x\| \geq \delta\|x\|$. Then $A(z)^{*} A(z) \geq \delta^{2}$ and hence

$$
\delta^{2 n} \leq \operatorname{det}\left(A(z)^{*} A(z)\right)=\sum_{l_{1}<\cdots<i_{n}}\left|\operatorname{det} A_{i_{1} \cdots i_{n}}(z)\right|^{2},
$$


where $A_{i_{1} \cdots i_{n}}$ is the $n \times n$ submatrix of $A$. Since $\operatorname{det}\left(A(z)^{*} A(z)\right)$ is upper bounded, by the infinite corona theorem, there are $b_{i_{1} \cdots i_{n}} \in H^{\infty}$ such that

$$
\sup _{z \in D} \sum_{i_{1}<\cdots<i_{n}}\left|b_{i_{1} \cdots i_{n}}(z)\right|^{2}<\infty, \quad \sum b_{i_{1} \cdots i_{n}} \operatorname{det} A_{i_{1} \cdots i_{n}}=1 \quad \text { on } D .
$$

Thus we can construct a bounded holomorphic function $B(z)$ such that $B(z) A(z)=I$ in the same way as Fuhrmann [6]. The converse is trivial, so we can conclude the proof.

Theorem 3.8. Let $T$ be a contraction. Then $T$ is similar to $S_{n}$ if and only if $T^{*} \in B_{n}^{h}(D)$, and there is a holomorphic frame $\gamma_{1 w}, \ldots, \gamma_{n w}$ for $\operatorname{ker}\left(T^{*}-w\right)$ and positive constants $M, \delta$ such that for any $x_{i} \in \mathbf{C}$ and $w \in D$

$$
M \sum_{i=1}^{n}\left|x_{i}\right|^{2} \geq\left(1-|w|^{2}\right)\left\|\sum_{i=1}^{n} x_{i} \gamma_{i w}\right\|^{2} \geq \delta \sum_{i=1}^{n}\left|x_{i}\right|^{2} .
$$

Proof. We use the notations in the proof of Theorem 3.3. Let $Y$ be an invertible operator satisfying $Y T=S_{n} Y$. Then $\gamma_{i w}=Y^{*} e_{i} /(1-w z)$ satisfies (3.1). It is clear that $T^{*}$ is in $B_{n}^{h}(D)$. Thus we must only show "if" part. We represent $\gamma_{i w}$ as (2.4), and determine $\Gamma(z): F_{2} \rightarrow C_{n}$ by $\Gamma(z) y=\sum_{i=1}^{n}\left(y, \gamma_{i \bar{z}}(0)\right) e_{i}$. Then we have $\Gamma^{\sim}(z) x=\sum_{i=1}^{n}\left(x, e_{i}\right) \gamma_{i z}(0)$ for $x \in \mathbf{C}_{n}, z \in D$. Thus, since

$$
\begin{aligned}
\left\|\Gamma^{\sim}(z) x\right\|^{2} & =\left\|\sum\left(x, e_{i}\right) \gamma_{i z}(0)\right\|^{2} \\
& =\left(1-|z|^{2}\right)\left\|\sum\left(x, e_{i}\right) \gamma_{i z}\right\|^{2} \quad \text { for every } z \in D,
\end{aligned}
$$

applying Proposition 3.7, $\Gamma(z)$ has the bounded right inverse. Therefore we have $H^{2}\left(\mathbf{C}_{n}\right)=\Gamma H^{2}\left(F_{2}\right)=\Gamma H(\theta)$, because $\Gamma \theta=0$. Consequently $X$ given by $X h=\Gamma h$ is an invertible operator from $H(\theta)$ to $H^{2}\left(\mathbf{C}_{n}\right)$ satisfying $X T=S_{n} X$ (see the proof of Theorem 3.3). Hence the proof is complete.

We observe that we can substitute $\left(1-|w|^{2}\right) G(w)$ for the middle term of (3.1), where $G(w)$ is the Gram matrix of $\gamma_{i w}, \ldots, \gamma_{n w}$.

Proposition 3.9. The contraction $T$ is similar to the isometry if and only if $T$ satisfies one of the following equivalent conditions:

(a) there is a positive constant $\delta$ such that $\left\|T^{n} x\right\| \geq \delta\|x\|$ for $x$ in $H$.

(b) There is a power-bounded operator $B$ satisfying $B T=I$.

(c) There is a bounded operator $B$ such that $B T=I$ and for any $w$ in $D$ $\left(I-w B^{*}\right)^{-1}$ exists and $\sup _{w \in D}(1-|w|)\left\|\left(I-w B^{*}\right)^{-1}\right\|<\infty$

Proof. In [15], Sz.-Nagy and Foias showed that $T$ satisfies (a) if and only if $T$ is similar to isometry. (a) $\Leftrightarrow$ (b) is trivial. Moreover it is clear that (c) follows from similarity of $T$ and isometry, and its converse is able to be shown in the same way as Castern [3], by considering

$$
\sum_{n=1}^{\infty} r^{n} e^{i n t} B^{* n}+\sum_{n=1}^{\infty} r^{n} e^{-i n t} T^{* n}
$$

instead of $\sum_{n=-\infty}^{\infty} r^{n} e^{i n t} S^{n}$ on p. 191 of [3]. 
At the end of this section we remark that from the above proposition we can get conditions for $T$ to be similar to $S_{n}$. For instance it suffices to add $T \in C_{._{0}}$ and $\operatorname{dim} \operatorname{ker} T^{*}=n$ to each condition of the above.

Acknowledgements. I would like to thank the referee for pointing out many grammatical errors, and I am grateful to $\mathrm{K}$. Takahashi for reading my original paper and pointing out a few mistakes.

\section{REFERENCES}

1. V. T. Alexander, Construction operators quasisimilar to a unilateral shift, Trans. Amer. Math. Soc. 283 (1984), 697-703.

2. S. Bergman, The kernel function and conformal mapping, 2nd ed., Math. Surveys, no. 5, Amer. Math. Soc., Providence, R. I., 1970.

3. J. A. Van Casteren, A problem of Sz.-Nagy, Acta Sci. Math. 42 (1980), 189-194.

4. M. J. Cowen and R. G. Douglas, Complex geometry and operator theory, Acta Math. 141 (1978), 187-261.

5. __ Operators possessing an open set of eigenvalues, Colloq. Math. Soc. János Bolyai 35, North-Holland, Amsterdam, 1980, pp. 323-341.

6. P. A. Fuhrmann, On the corona theorem and its application to spectral problems in Hilbert space, Trans. Amer. Math. Soc. 132 (1968), 55-66.

7. G. M. Goluzin, Geometric theory of functions of a complex variable, Transl. Math. Monographs, Amer. Math. Soc., Providence, R. I., 1969.

8. H. Helson, Lectures on invariant subspaces, Academic Press, 1964.

9. G. Misra, Curvature inequality and extremal properties of bundle shifts, J. Operator Theory 11 (1984), 305-317.

10. __, Curvature and the backward shift operators, Proc. Amer. Math. Soc. 91 (1984), 105107.

11. M. Rosenblum, A corona theorem for countably many functions, Integral Equations and Operator Theory 3 (1980), 125-137.

12. K. Seddighi, Essential spectra of operators in $B_{n}(\Omega)$, Proc. Amer. Math. Soc. 87 (1983), 453-458.

13. A. Shields, Weighted shift operators and analytic function theory, Topics in Operator Theory, Math. Surveys, no. 13, Amer. Math. Soc., Providence, R. I., 1974, pp. 49-128.

14. B. Sz.-Nagy and C. Foiaş, Harmonic analysis of operators on Hilbert space, North-Holland, 1970.

15. B. Sz.-Nagy and C. Foias, On contractions similar to isometries and Toeplitz operators, Ann. Acad. Sci. Fenn. 2 (1976), 553-564.

16. K. Takahashi, On quasiaffine transform of unilateral shifts, Proc. Amer. Math. Soc. 100 (1987), 683-687.

17. M. Uchiyama, Contractions and unilateral shifts, Acta Sci. Math. 46 (1983), 345-356.

18. Contractions with $(\sigma, c)$ defect operators, J. Operator Theory 12 (1984), 221-233.

19. R. O. Wells, Differential analysis on complex manifolds, Prentice-Hall, Englewood Cliffs, N. J., 1973.

20. P. Y. Wu, Contractions quasisimilar to an isometry, preprint.

Department of Mathematics, Fukuoka University of Education, Munakata, Fukuoka, 811-41 JAPAN 